\title{
Nutritional contributions to dementia prevention: main issues on antioxidant micronutrients
}

\author{
Maria Cristina Polidori • Ralf-Joachim Schulz
}

Received: 28 August 2013/Accepted: 23 December 2013/Published online: 18 February 2014

(C) Springer-Verlag Berlin Heidelberg 2014

\begin{abstract}
There is an impressing body of evidence supporting the beneficial role of balanced nutrition in lowering the risk of dementia and its commonest form, Alzheimer's disease. Nevertheless, and despite worldwide dementia epidemic, there is much unfounded skepticism and lack of information among physicians. As a result, the diagnosis of cognitive impairment occurs still far too late, at best symptomatic drugs keep being prescribed and patients and caregivers are left with little concrete support in the hands of the natural history of the disease. This review summarizes knowledge about the impact of nutrition as part of a healthy lifestyle and of micronutrients in particular on delaying and avoiding dementia onset.
\end{abstract}

Keywords Nutrition - Clinical trial · Antioxidant · Oxidative stress $\cdot$ Alzheimer's disease $\cdot$ Mild cognitive impairment

\section{Why do we need to care about dementia}

Dementia is the most prevalent neurodegenerative disorder, and it is characterized by progression, multiple etiology, complex symptomatology including that related to a disturbance of cognitive performance and absence of a cure. During the course of the disease, the cognitive symptoms of dementia persist and steadily worsen and behavioral

This paper is part of Genes and Nutrition's “Topical Collection on Ageing."

M. C. Polidori ( $\square)$ · R.-J. Schulz Department of Geriatric Medicine and Research, St. Marien-Hospital and University of Cologne, Kunibertskloster 11-13, 50668 Cologne, Germany e-mail: maria.polidori-nelles@uk-koeln.de disturbances appear and progress and as a result intellectual and social abilities are affected enough to obstacle the performance of activities of daily living. According to dementia definition, a diagnosis of dementia can be done when the impairment of more cognitive domains lasts long enough and is associated with behavioral disturbances both affecting activities of daily living. Dementia is indeed associated with disability and a high grade of dependence on caregivers-usually family members. This condition of disability and dependence brings along an enormous social and economic burden which is linked to the epidemics of dementia and its commonest, untreatable form, Alzheimer's disease (AD). The major risk factor for dementia and $\mathrm{AD}$ is advanced age, and therefore, the societal impact of $\mathrm{AD}$ is increasing according to aging demographics (http:// ec.europa.eu/health/reports/european). Life expectancy at birth in the EU increased from 72 years in 1980 to 78 years in 2007. The population of industrialized countries progressed from a mean life expectancy of 35 years at the beginning of the last century, to currently over 80 years of age. People aged 65 years or older are expected to double between 1995 and 2050 to reach 135 million in the EU, with very old people, aged 80 years and older, being projected to grow as much as eight to ten times on the global scale by 2050 . This will strongly impact the already challenging number of ten million $\mathrm{AD}$ cases in the EU. As a cure against dementia has not been found yet, health care professionals all over the world are predicted to face the diagnostic, therapeutic and socioeconomical challenges of over 115 million people with dementia by 2050 (Prince et al. 2013). This perspective might be even underestimated, particularly due to inadequate diagnosis, lack of awareness and low education. The dramatic loss of synapses and cholinergic neurons, accumulation of extracellular $\beta$ amyloid $(\mathrm{A} \beta)$ plaques and intraneuronal fibrillary 


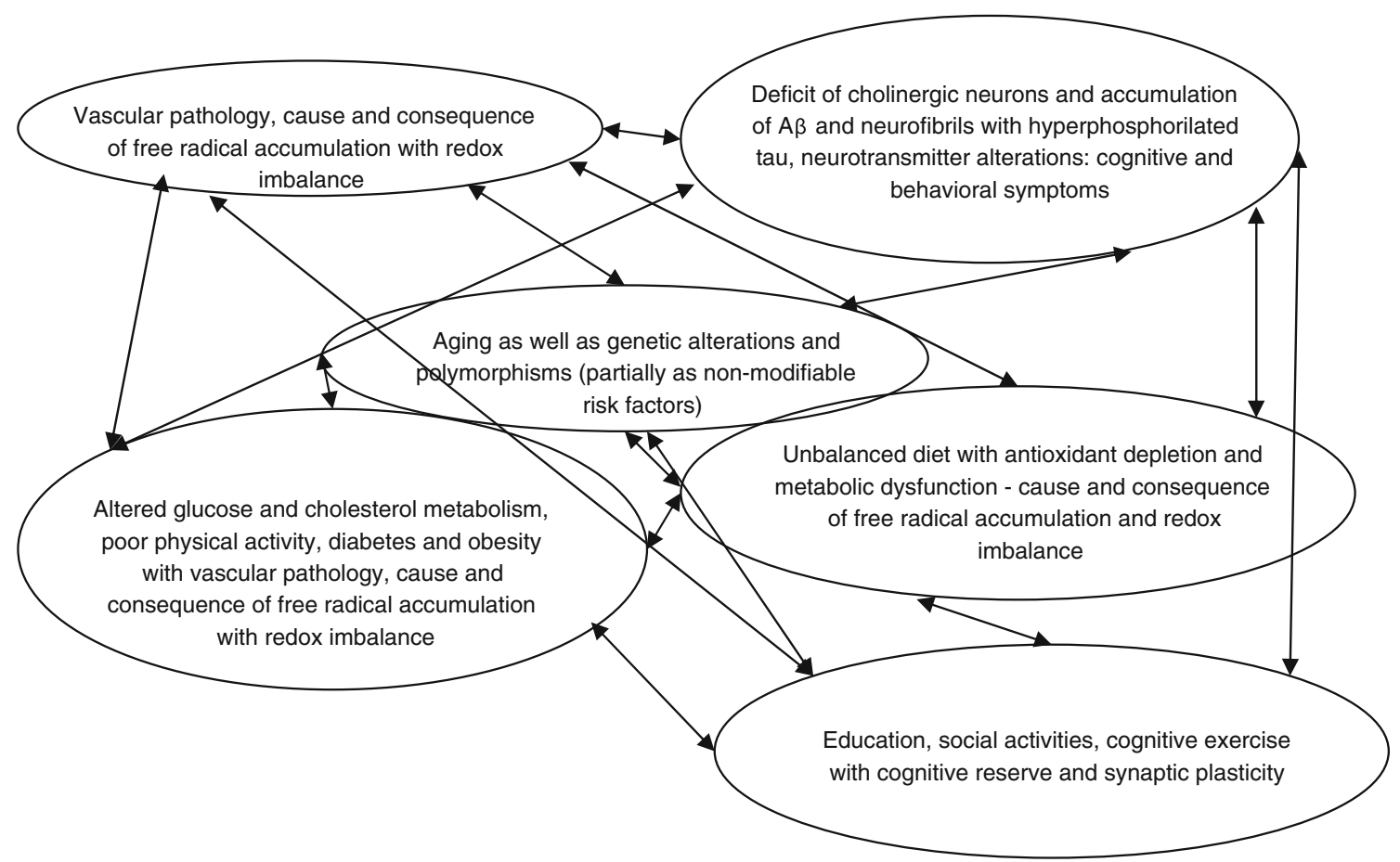

Fig. $1 \mathrm{AD}$ is a complex of symptoms with multiple causes and interrelated influencing factors

tangles of hyperphosphorylated tau are the major hallmarks of $\mathrm{AD}$ brain and are implicated in its pathogenesis. However, the nature of $\mathrm{AD}$ is multifactorial, as a direct consequence the clinical disorder is complex (Fig. 1) and a cure against it is difficult to find. In spite of the earlier, decade-lasting stratification of dementia cases in vascularversus AD-type, there is accumulating evidence that vascular pathology plays a central role in $\mathrm{AD}$ onset and development (Polidori and Pientka 2012; Polidori et al. 2012), and some important information from recent reanalyses includes data from a 75+ year-old community cohort in which $49 \%$ of the clinically diagnosed AD cases showed a possible vascular component (Agüero-Torres and von Strauss 2006). On the other hand, the next most prevalent dementia form, vascular dementia, presents overlapping traditional hallmarks of $\mathrm{AD}$ including $\mathrm{A} \beta$ accumulation. While the vascular component of $\mathrm{AD}$ is currently matter of great attention, AD's etiology remains unclear and neither genes alone nor environment alone is sufficient to explain disease onset. For these reasons, the translation of "clean" bench experiments on cell cultures and animal models into bedside studies on elderly demented patients with multimorbidity and on multitherapy is a highly demanding one.

Recently, Larson et al. (2013) highlighted the decline of age-specific prevalence or incidence rates of dementia reported in several recent studies on subjects born later in the first half of the twentieth century. The epidemiologic data from European and American studies are strongly suggestive of the beneficial effects of higher levels of education, reduction in stroke rates, the overall ameliorating trends in medical, lifestyle and social factors. In the Rotterdam Study, larger brain volumes and less extensive cerebral small-vessel disease on magnetic resonance imaging in persons born later were shown (Schrijvers et al. 2012). Even though the projected growth of the population older than 75 years guarantees a growing epidemic of dementia, the results of the aforementioned studies indicate that individual vascular and other risk factor control is likely to produce substantial public health benefits through dementia risk reduction. There is a large body of evidence on the beneficial effects of a healthy lifestyle including physical exercise and vascular risk control against AD onset and development which has been recently extensively summarized (see the 2012 special issue of the Zeitschr. Gerontol Geriatr and European Journal of Geriatrics on dementia and of the Journal of Alzheimer's Disease, Physiopathology of vascular risk factors in Alzheimer's Disease; Mangialasche et al. 2010). Their recapitulation would be redundant and goes beyond the scope of this special section. In this review, specific aspects of nutrition during aging and $\mathrm{AD}$ will be put into the perspective of their potential role in modulating cognitive impairment with and without dementia. A detailed inventory of all antioxidant molecules tested or being tested in in vitro, in vivo, ex vivo experiments in animal models and humans has been published in different constellations elsewhere (2012 special issue of BioFactors, Biofactors 
and cognitive functions; Mecocci and Polidori 2012; Polidori and Nelles 2013). The aim of this review is to present evidence on nature, possible mechanisms of action and effects of adequate levels of micronutrients as part of a balanced nutrition against the AD-related detrimental pathophysiology of neurodegeneration in the elderly.

\section{Why can't we stop searching for oxidative stress-related mechanisms of $A D$ pathophysiology}

Among the mechanisms identified to play a major role in the continuum between aging brain, cognitive impairment and $\mathrm{AD}$ and other dementia forms, the overproduction of reactive oxygen and nitrogen species (ROS and RNS, respectively) as well as of other free radical species has been shown to occupy a central position. In the absence of an adequate antioxidant defense system, ROS and RNS damage to body lipids, nucleic acids and proteins has been consistently shown to be associated with the presence of AD-related alterations and symptoms as well as to disease severity. By-products of oxidative stress and redox unbalanced regulation as well as antioxidant depletion independent of diet have been repeatedly observed in plasma and tissues of patients with cognitive impairment even long prior to overt dementia manifestation (Praticó et al. 2002; Rinaldi et al. 2003) suggesting chronological primacy of oxidative stress in AD (Smith et al. 2005; Yan et al. 2013). The involvement of oxidative stress at different levels in $\mathrm{AD}$ in neuronal mitochondria is particularly deleterious due to their susceptibility to oxidative damage. Mitochondria undergo a series of oxidation-mediated events during aging and neurodegeneration (Leuner et al. 2012). Mitochondrial DNA oxidative changes lead to alterations of form and function, and aggregation of proteins as well as lipid peroxidation and membrane dysfunction. The compromised mitochondrial respiration is associated with sustained hypoperfusion promoting in turn oxidative stress of brain tissue. This causes secondary damage via the overexpression of inducible and neuronal-specific nitric oxide synthases and reduced glucose utilization. A permanent accumulation of oxidative stress products may sustain a vicious circle in which brain damage is accelerated and the blood brain barrier is compromised. Altered cellular metabolism and oxidative stress within AD-related cerebral hypoperfusion, in the presence of vascular risk factors, might favor the reach of a critical threshold for cerebral hypoperfusion (de la Torre 2000, 2013). The resulting increase in oxidative stress might damage the neurovascular unit and the endothelium of brain vessels thereby favoring $A \beta$ formation and nourishing a vicious circle in which plaque-induced ROS formation also damages the neurovascular unit through the endothelium (Vagnucci and
Li 2003). ROS and other free radical species production is an early event in AD development, and the disturbed balance between oxidant and antioxidant production has been shown to be associated with the severity of cognitive impairment even in the absence of dementia or with the degree of cognitive performance in healthy subjects across a wide of age range. A large amount of studies since the definition of oxidative stress (Sies 1985) and antioxidants (Sies 1993) have been conducted with the aim of identifying oxidation-lowering strategies capable to reduce AD clinical manifestation and improve cognitive performance. Trials aimed at exploring the efficacy of antioxidant strategies in $\mathrm{AD}$ and in what is considered its preclinical form, mild cognitive impairment (MCI), however, have been largely unsuccessful so far (Mecocci and Polidori 2012; Polidori and Nelles 2013). A section of this review is dedicated to the several reasons accounting for this lack of success. Briefly, antioxidant trials against AD have been designed in all these years like any other clinical trial using antidementia and regardless of the biochemical mechanistic peculiarity of antioxidant/micronutrient use in aged people with dementia. The lack of efficacy of single antioxidant micronutrients at an advanced AD stage does not disprove the role oxidative stress in AD onset and development. In contrast, the negative results available should be taken into account to improve further research on AD pathophysiology.

\section{Why antioxidant pathways against cognitive impairment should be further explored}

For dementia risk control-similar to maintenance of healthy aging - the attention of researchers, clinicians and industries has sharply converted in the past few decades on single antioxidant supplements. However, cellular protection against oxidative toxicity is provided by a large amount of molecules of different size, all of them interacting in a network, acting synergistically and strongly dependent in level and function on the overall biochemical balance of the organism. Endogenous antioxidants are mainly represented by glutathione (GSH) and antioxidant enzymes such as GSH peroxidase, catalase and superoxide dismutase. Small-molecule antioxidants may act directly as redox active, short-lived compounds which need to be regenerated or replenished after consumption, or indirectly, are not always redox active but induce transcriptionally several cytoprotective proteins, or phase- 2 enzymes, that act catalytically, live longer and are not consumed. Direct antioxidants remove and scavenge excess ROS and their precursors, inhibit formation of excess ROS and other free radicals and bind metal ions necessary for the catalysis of excess ROS generation. Antioxidant molecules such as the 
exogenous vitamin $\mathrm{C}$, vitamin $\mathrm{E}$ and carotenoids among other direct antioxidants have been used in the therapy of MCI and AD. On the basis of the plethora of studies showing low micronutrient and vitamin intakes and plasma levels in AD subjects (Masaki et al. 2000; Engelhart et al. 2002; Morris et al. 2002; Grodstein et al. 2003; Larrieu et al. 2004), even prospectively (Morris et al. 2005a, b), several single antioxidant molecules have been tested in different $\mathrm{AD}$ models with the aim of neutralizing free radicals. A recent meta-analysis of seven studies on dietary intakes of vitamin $\mathrm{E}$, vitamin $\mathrm{C}$ and $\beta$-carotene confirmed a relative risk for developing $\mathrm{AD}$ of 0.76 for vitamin $\mathrm{E}, 0.83$ for vitamin $\mathrm{C}$ and 0.88 for $\beta$-carotene (Feng-Jiao et al. 2012). The evidence of an association between antioxidant status and dementia has only partially been confirmed in the Cache County Study, in which no protective effect of vitamin $\mathrm{C}$ or vitamin $\mathrm{E}$ alone was shown against $\mathrm{AD}$ onset (Zandi et al. 2004). Also the Washington Heights-Inwood Columbia Aging study (Luchsinger et al. 2003) failed to show protection of vitamin $\mathrm{C}$, vitamin $\mathrm{E}$ or carotenes against $\mathrm{AD}$ risk. Similar observations were obtained in the Women's Health Study (Dumont et al. 2010) and the AgeRelated Eye Disease Study (Yaffe et al. 2004). The major problem common to all these studies is the difficult interpretability and consequent comparability with the results of similar trials. Studies after the pioneer ones have been designed without taking into account homogeneity and numerousness of tested groups as well as type and dose of supplement, endpoint and biomarkers. In other words, study designs have been not substantially ameliorated despite the identification of weaknesses of previous observations.

The inconsistencies between observational and longitudinal studies on antioxidant intakes against $\mathrm{AD}$ risk have been found partly simultaneously with the performance of a pivotal trial using vitamin $\mathrm{E}$ in $\mathrm{AD}$ patients. Vitamin $\mathrm{E}$ is the most studied molecule in AD (Joshi and Praticó 2012). In the trial of vitamin $\mathrm{E}$ on 341 patients with moderate-tosevere AD (Sano et al. 1997), patients treated with a daily dose of 2,000 IU of vitamin E for 2 years showed a significant delay in $\mathrm{AD}$ progression and in nursing home placement compared to patients treated with deprenylwhich inhibits the free radical-generating degradation of catecholamines by monoamine oxidase B-or placebo subjects. No effect was observed on Mini-Mental State Examination score at the end of the study. The results could up-to-date not be readily interpretable due to an unfortunate failure of randomization, with the placebo group at baseline scoring significantly better cognitively than the other groups (Sano et al. 1997). In another study from the Alzheimer's Disease Cooperative Study which had conducted the vitamin $\mathrm{E}$ trial in AD patients, 769 subjects with MCI, the clinical phase preceding AD, were randomly assigned to receive 2,000 IU of vitamin E daily or $10 \mathrm{mg}$ of donepezil or placebo for 3 years (Petersen et al. 2005). At the end of the study, 212 developed AD, with $16 \%$ conversion rate per year. The hazard ratio indicating the probability of progression to $\mathrm{AD}$ in the vitamin E group was 1.02, not significantly different from that of donepezil (0.80). There were no significant differences in the rate of progression to $\mathrm{AD}$ between vitamin $\mathrm{E}$ and placebo groups at any point, either considering all patients or the apolipoprotein E $\varepsilon 4$ carriers only. The secondary outcome measures of the study included changes of cognition as assessed by MMSE, CDR sum of boxes, Global Deterioration Scale and modified ADAS-Cog which showed few weak significant differences in the vitamin E group compared with the placebo group (Petersen et al. 2005). The lack of clear-cut results is mainly due to the problematic use of a single compound against a multi-mechanism disease which is already clinically overt. There are also a large number of biochemical and metabolic factors that have not been cleared yet. The role of metabolites of antioxidants compounds selected for treatment might have a large number of yet unknown biological actions. The same is valid for the antioxidants themselves. Intra- and interindividual responses to antioxidant supplementation may strongly influence data analysis. In addition, negative findings of vitamin E supplementation trials may arise in part from the not-contemplated depletion of $\gamma$ tocopherol that occurs when high-dose $\alpha$-tocopherol supplements are taken (Hensley et al. 2004). Influences of a given antioxidant supplement on the antioxidant network of the organism have not been evaluated in the above cited trials.

Vitamin E supplement use against dementia is the paramount example of antioxidant supplementation with the intention to treat dementia. The results of both trials (in AD and MCI patients) were not encouraging as far as clinical amelioration is concerned. This information adds to the poor comparability of other studies, of epidemiological, observational or cohort nature, focusing on individual antioxidants against cognitive impairment. Nevertheless, the majority of the ongoing antioxidant clinical trials against MCI and AD still use single supplements with antioxidant potential. Two most recent clinical trials with vitamin $\mathrm{E}$ in $\mathrm{AD}$, the Prevention of Alzheimer's Disease by Vitamin E and Selenium (PREADVISE) [clinicaltrials.gov NCT00040378] and the TEAM-AD, a randomized, clinical Trial of vitamin E And Memantine in Alzheimer's Disease [clinicaltrials.gov NCT00235716], are in the analysis phase.

Several other clinical trials with single substances displaying antioxidant properties such as polyphenols with curcumin, flavonoids and resveratrol are active. Importantly, some of these substances act as the indirect 
antioxidants described above, induce cytoprotective proteins or phase- 2 enzymes and have been isolated from edible plants, e.g., curcumin from turmeric. Curcumin is being evaluated in one phase II trial of 2 or $3 \mathrm{~g}$ per day of curcumin versus placebo in patients with moderate-tosevere AD treated for 60 days [clinicaltrials.gov NCT01001637] and in subjects with MCI treated or not with curcumin for 18 months with the aim of evaluating amyloid deposition in the brain with FDDNP-PET scan [clinicaltrials.gov NCT01383161]. There is an ongoing phase II/III RCT on 50 patients with early AD already under donepezil treated with epigallocatechin gallate versus placebo for 18-month recruitment phase. The primary endpoint of the study is the ADAS-cog score at study end [clinicaltrials.gov NCT00951834]. Resveratrol is under investigation versus placebo in a RCT using liquid resveratrol with glucose and malate as a dietary supplement delivered in grape juice in $60 \mathrm{AD}$ patients with the primary endpoint of assessing ADAS-cog scores at regular intervals up to 1 year after study commencement [clinicaltrials.gov NCT00678431]. Resveratrol is also being studied in MCI patients cognitively assessed by means of ADAS-Cog [clinicaltrials.gov NCT01219244].

The family of mitochondrial antioxidants is also under active investigation, due to the fact that neuronal mitochondria are particularly susceptible to oxidative damage. These molecules include pramipexole, latrepirdine, ubiquinone, acetyl-L-carnitine and R-alpha-lipoic acid. Pramipexole is being studied in an ongoing phase II trial of 20 patients with $\mathrm{AD}$ treated with R-pramipexole twice a day for a total dose of $100 \mathrm{mg}$ per day. After 4 weeks, the dose is doubled and after another 4 weeks, patients are treated with $300 \mathrm{mg}$ R-pramipexole per day that is maintained for the subsequent 16 weeks. There is no placebo arm, and the primary endpoints are safety issues and effects on cognitive performance, while secondary outcome measures are lowering of oxidative stress as assessed by CSF isoprostanes levels before and after treatment as well as changes in cerebral glucose metabolism as assessed by PET scan before and after treatment. The estimated study completion date is April 2014 [clinicaltrials.gov NCT01388478]. Although a phase III trial with latrepirdine showed no cognitive effects after 26 weeks of treatment in $\mathrm{AD}$ patients, there is an ongoing phase III multicenter, randomized, placebo-controlled, double-blind 12-month safety and efficacy study with latrepirdine versus placebo in patients with mild-to-moderate AD on donepezil (Concert study) with ADAS-cog and ADCS-ADL as primary endpoints [clinicaltrials.gov NCT00829374]. The effects of another mitochondrial antioxidant, ubiquinone, are being evaluated and analyzed after the performance of a phase I investigation to evaluate the safety, tolerability and impact on biomarkers of antioxidant treatment of mild-to- moderate AD. This trial started in 2006 in the frame of the Alzheimer's Disease Cooperative Study and included 75 patients with $\mathrm{AD}$ who underwent treatment with either coenzyme Q $400 \mathrm{mg}$ or vitamin E 800 IU plus vitamin C $200 \mathrm{mg}$ plus $\alpha$-lipoic acid $600 \mathrm{mg}$ or placebo three times a day [clinicaltrials.gov NCT00117403]. The primary endpoints were changes in CSF biomarkers after 4 months therapy, and secondary outcome measures were the CSF levels of $\beta$-amyloid 40 and $\beta$-amyloid 42 . No results of this trial are available up-to-date. Lipoic acid supplementation reduces memory loss and appears to stabilize cognitive function. Lipoic acid is being tested in a phase I/II RCT. The latter is ongoing and will include $100 \mathrm{AD}$ patients treated with $600 \mathrm{mg}$ lipoic acid plus $3 \mathrm{~g}$ fish oil concentrate or with placebo for 18 months [clinicaltrials.gov NCT01058941].

Basically, there is still a big debate on how the wealth of evidence in favor of adequate antioxidant intakes and levels against dementia development might be depending upon the natural versus synthetic source of antioxidants. In addition, it appears that after the dissemination of negative study results, especially of those interventional ones, little attention is being paid to the eventual limitations of study designs. This happens even in spite of a large amount of literature assessing and carefully reviewing these limitations. RCTs performed so far have clearly shown that single antioxidant compounds are extremely unlikely to display beneficial effects against cognitive impairment progression in patients with overt MCI and AD. Nevertheless, new RCTs with single antioxidant compounds are kept being performed and designed for investigation against disease progression. The inconsistency of data obtained so far should not prevent from further search of appropriate trial design in dementia as well as of biomarkers of antioxidant activity in humans and of antioxidant interactions and profiles specifically able to counteract neurodegenerative pathways in $\mathrm{AD}$.

\section{Why randomized controlled trials with antioxidants against MCI and AD are unsuccessful}

Figure 2 summarizes the major flaws of unsuccessful clinical trials of antioxidants in AD. Each of the past and ongoing randomized controlled trials described above utilizes a different single compound against MCI or AD. None of the substances tested against MCI and AD showed a clear, readily interpretable, consistently reproducible benefit. A very few studies testing the effects of a combination of two substances or antioxidant mixtures also display little benefit against $\mathrm{AD}$ onset or progression as well as against the transition of MCI to AD. All critical components of these trials, from sample size to cognitive measures used, are not comparable. Diagnostic criteria of 


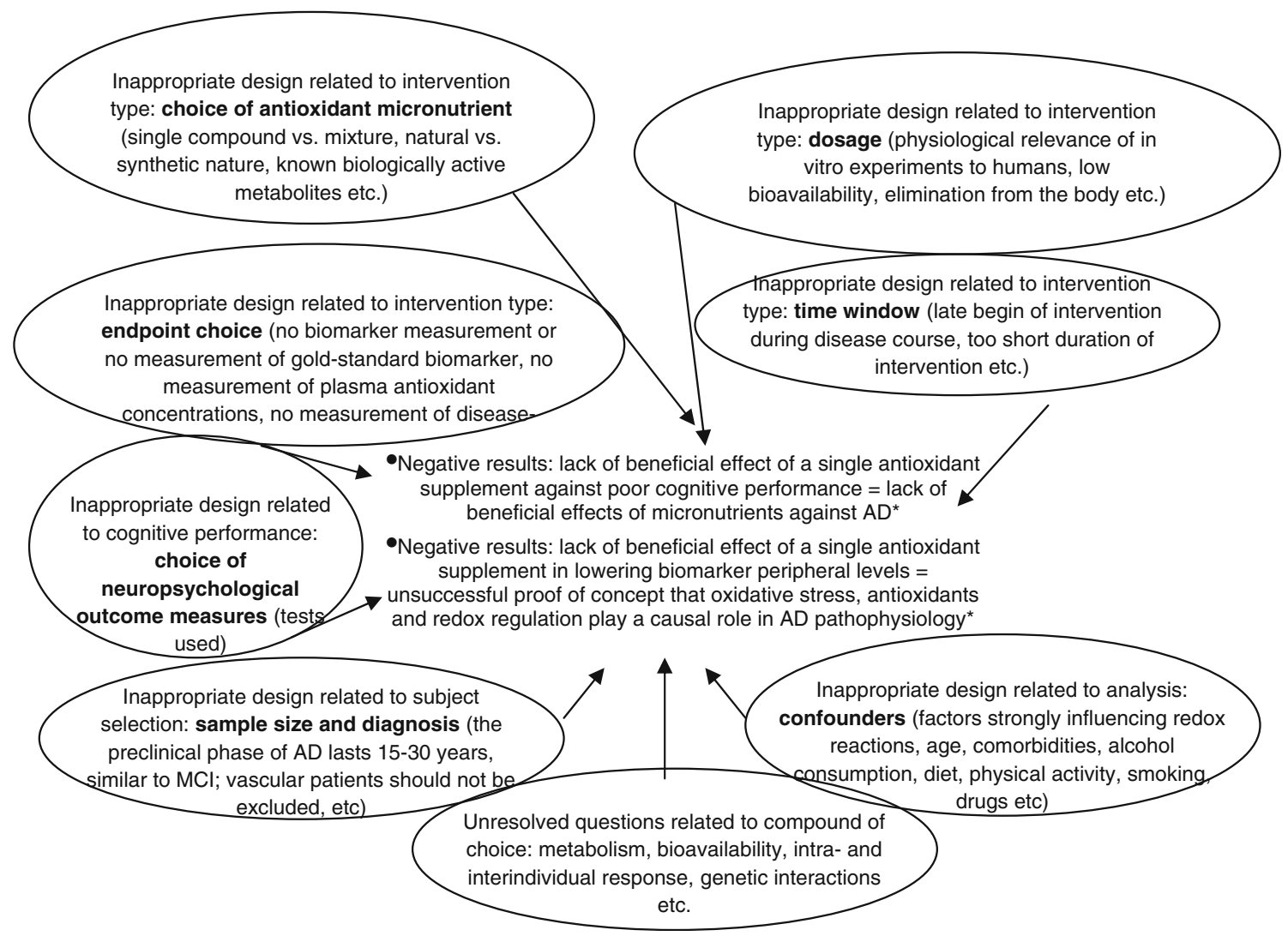

Fig. 2 Major flaws of unsuccessful clinical trials of antioxidants in AD. *Common misleading equation between unadulterated result of the trials and its interpretation as far usefulness of the oxidative stress-antioxidant balance in AD is concerned

MCI and AD (Whitehouse and George 2011) as well as inclusion criteria and outcomes for these studies are at best not homogeneous, so that very different patients have been studied in different trials. This applies particularly to patients with MCI which may be possibly classified as controls and to mild AD patients which might be diagnosed as MCI. Furthermore, AD patients with cardiovascular risk factors or comorbidities have been misdiagnosed for many years and excluded from AD studies. Start and end of the intervention with a particular antioxidant in patients with $\mathrm{MCI}$ or $\mathrm{AD}$ have been set for logistic reasons mostly so that intervention begin occurs too late during the course of the disease and study end delimits a far too short intervention period. In this sense, the probability that even the best available biomarker of free radical-induced molecular damage will not display any major changes at the end of the study reshapes criticism against lack of biomarker use in RCTs. There is also a number of specific compoundrelated issues likely responsible for the lack of trial success, including dosage-, kinetic-, bioavailability-, metabolic and genetic-related issues that have been repeatedly discussed (http://lpi.oregonstate.edu/infocenter/cognition. html). Despite this, it seems difficult to uniform trial design and method in $\mathrm{AD}$ nutritional studies.

\section{Why should we care about diet and nutrition against cognitive impairment}

The brain relies on the presence of adequate amounts of nutrients for formation and maintenance of nerve cells and neurotransmitters. Both macro- and micro-nutrients provide biomolecules and precursors whose unique source for proper functioning is natural nutrition. Among natural sources of protective substances like vitamins and antioxidant micronutrients, fruits and vegetables play a major role. Epidemiological research has been conducted in the past two decades to address the status and effects of fruit and vegetable intake in several populations. As expected (Hippocrates, V century BC), high daily intake of fruits and vegetables is integrative part of a healthy lifestyle (Lambrinoudaki et al. 2013). Interestingly, the term "diaeta" was adopted by the Romans from the word " $\delta i \alpha \dot{\eta} \tau \alpha$ " with which the ancient Greeks intended the "way of living," not only nutrition. To date, there is sufficient evidence to state that the burden of disease attributable to poor nutrition in Europe is substantial (Pomerleau et al. 2003; Boeing et al. 2012) and that a poor diet is one of the leading causes of death for Europeans (http://ec.europa.eu/health/ reports/european). According to the European Nutrition 
and Health Report (Elmadfa 2009) and to the European Food Information Council (http://www.eufic.org/article/en/ expid/Fruit-vegetable-consumption-Europe/), most of the European countries do not meet the recommendations of daily fruit and vegetable intake by the WHO, with large gradients of intake across countries.

The aging organism bases on a closer correlation between dietary habits, nutritional intakes and plasma micronutrient status than younger subjects. Elderly subjects, and in particular elderly individuals with dementia and AD, have decreased food intakes, in most of the cases eating behavior disturbances, and loss of body weight. The effects of aging demographics on disabling disease are being currently matter of great public health-related attention, as stated in the latest WHO global brief for World Health Day. The brief also highlights that dementia is the first cause of years lost due to disability in highincome countries and the second greatest worldwide (http://whqlibdoc.who.int/hq/2012/WHO_DCO_WHD_ 2012.2_eng.pdf). Nutrition and dementia, therefore, are the leading public health concerns in Europe, with nutrition also strongly influencing a large number of risk factors and comorbidities of dementia and AD including vascular ones (Polidori et al. 2012). While nutrition is a wide and complex field including aspects of caloric restriction, fat and alcohol consumption as well as fiber and cereal intake and weight maintenance, suboptimal micronutrient status is an early feature in the onset of AD (Ames 2006a, b; extensively reviewed in the second issue of the 38th volume of BioFactors dedicated to cognitive function, Polidori 2012a). Four cohort studies have been conducted in the past decade, which collectively suggest an inverse relation between risk of dementia and vegetable and fruit consumption. Over 1,800 Japanese immigrants were assessed with respect to $\mathrm{AD}$ incidence up to 9 years after a survey about intake of fruit and vegetable juices (Dai et al. 2006). In this study, a lower disease incidence with increased juice intake was observed and the finding was independent of vitamin C, E and $\beta$-carotene intake (Dai et al. 2006). A prospective cohort study of over 3,700 older participants of the Chicago Health and Age Project showed an association of high vegetable consumption with a slower rate of cognitive decline over 6 years after adjusting for age, gender, race, education, cardiovascular-related conditions and risk factors (Morris et al. 2006). In this study, the consumption of green leafy vegetables, rich in antioxidant micronutrients like carotenoids, showed the strongest inverse linear association with the rate of cognitive decline. The specific protection shown by vegetables and particularly by the green leafy ones appears to be in disagreement with the concept that fruit and vegetable consumption might be beneficial in the frame of a generally healthy lifestyle, as health-conscious individuals tend to consume both fruits and vegetables. A positive association between fruit and vegetable intake, plasma antioxidant micronutrient level and cognitive performance was observed recently in 193 healthy subjects aged 45-102 years (Polidori et al. 2009a). In this study, lycopene, a powerful antioxidant carotenoid, was associated with several measures of cognitive performance. Previous studies showed that levels of circulating carotenoids, among other micronutrients, are associated with fruit and vegetable intake in the healthy elderly (Anlasik et al. 2005). In addition, plasma levels of carotenoids and other micronutrients might be increased through a nutritional interventional program with high intakes of fruits and vegetables (Polidori et al. 2009b). Therefore, in light of the epidemiological relevance of green leafy vegetable intake in cognitive deterioration (Morris et al. 2006), nutritional counseling programs in patients at risk for $\mathrm{AD}$ might prove beneficial. The beneficial role of fruit and vegetable intake on dementia incidence also include the HARMONY Study, a cohort from the Swedish Twin study (Hughes et al. 2010), the ESPRIT Study (Ritchie et al. 2010) and the Three-City Cohort Study (Barberger-Gateau et al. 2007). In the latter study, frequent fruit and vegetable consumption but also consumption of fish and $\omega-3$ fat was associated with a lowered risk of dementia and of $\mathrm{AD}$ especially among ApoE $\varepsilon 4$ noncarriers (Barberger-Gateau et al. 2007). The study showed a dementia risk reduction by about $30 \%$ in daily consumers compared to subjects rarely consuming fruits and vegetables. The HARMONY study demonstrated a significant risk reduction for AD development in subjects assuming high amounts of fruit and vegetables compared to poor consumers in midlife followed up over 30 years (Hughes et al. 2010). In the Esprit study, consumption of $<2$ portions fruits and vegetables per day was associated with a significantly higher risk of MCI and dementia compared to subjects assuming more than two portions (Ritchie et al. 2010).

As mentioned above, a balanced diet-as part of a healthy lifestyle_rather than single antioxidant molecules is the pathway to the delay of the onset of $\mathrm{AD}$ and several other age-related diseases. Antioxidant nutrients alone cannot account for the preventive effects of whole healthy nutrition and fruit and vegetable intake against dementia. Fruits and vegetables are thought to represent the best source of antioxidant micronutrients due to the shown synergisms of their components. Protective compounds from fruits and vegetables including, but not limited to, antioxidant micronutrients show a high bioavailability compared to single vitamins. Fruits and vegetables are also poor in saturated fat and Mediterranean diets contain saturated fat corresponding to $8 \%$ or less of daily calorie allowance (Willett et al. 1995). $\omega-3$ Polyunsaturated fatty acids (PUFAs) in general and long-chain $\omega-3$ PUFAs 
[eicosapentaenoic acid (EPA) and docosahexaenoic acid (DHA), the most abundant long-chain polyunsaturated fatty acid in the brain] in particular have beneficial effects on cardio- and cerebro-vascular function, including antiinflammatory, antiarrhythmic, antiaggregant and antiatherogenic effects (Friedland 2003). These PUFAs are object of active interest in AD research (Polidori et al. 2012) and are interestingly a main component of Mediterranean dietary patterns, per se shown to be inversely related to the risk of developing AD (Scarmeas et al. 2006, 2009a, b). On the other hand, brain cholesterol metabolism has been shown to play a pivotal role in dementia onset and worsening. Excess cholesterol is part of a vicious circle in which cholesterol-dependent amyloid deposition causes neuronal death and the consequent myelin breakdown increases brain cholesterol levels (Hughes et al. 2013). An interesting investigation by Bayer-Carter et al. (2011) showed that a diet low in saturated fats and simple sugars or a high-saturated fat, high-sugar diet for a month causes significant and opposing changes in several CSF biomarkers including dramatic changes in A $\beta 42$ in elderly subjects.

During the years, the beneficial role of a balanced diet rather than of a high intake of fruits and vegetables alone has been confirmed. The dietary regimen mostly studied and with the highest scientific evidence for being protective against dementia onset is the Mediterranean diet. Fruits and vegetables constitute a substantial part of the Mediterranean diet. As already highlighted by Willet in the mid1990 s, this diet is rich in plant-based food with fresh fruits as the typical daily dessert and olive oil as the principal source of fats (Willett et al. 1995). The Mediterranean diet is a diet characterized by high intake of fish, vegetables, legumes, fruits, cereals, unsaturated fatty acids mostly in the form of olive oil, low intake of dairy products, meat and saturated fatty acids as well as a regular but moderate intake of alcohol. The total fat in this diet is 25-35\% of daily calorie allowance with saturated fat at $8 \%$ or less of daily calorie allowance (Willett et al. 1995). Several studies have shown that adherence to a Mediterranean diet is associated with lower early death rates (Knoops et al. 2004) as well as with lower risk for developing cardiovascular disease (de Lorgeril et al. 1999) and diabetes (Martínez-González et al. 2008); the Lyon Diet Heart Study was the first RCT to show that a Mediterranean diet style helps reduce recurrent events in patients with heart disease (de Lorgeril et al. 1999; Kris-Etherton et al. 2001). After this study, the incidence of several diseases was evaluated in relation to the adherence to the Mediterranean diet. Scarmeas et al. reported in the past few years that adherence to this dietary profile is associated with a risk reduction for MCI (Scarmeas et al. 2009a) and AD (Scarmeas et al. 2006), independently of physical exercise
(Scarmeas et al. 2009b). Shortly prior to these clarifying observations on Mediterranean diet and cognitive impairment, Sofi et al. (2008) had conducted a meta-analysis of eight prospective studies and showed a strong overall association of Mediterranean diet adherence with a significant reduction in overall mortality and mortality for cardiovascular disease as well as with a primary prevention of cancer, Parkinson's disease and AD (13\% risk reduction). A review of 34 studies in the areas of dietary restriction, antioxidants and Mediterranean diet provided evidence that nutritional interventions against dementia and $\mathrm{AD}$ have a great potential of influencing dementia development (Burgener et al. 2008).

\section{Reflection points for future research}

Dementia has been proven to be a mostly heterogeneous age-related condition against which no individual preventive strategy and no single curative drug have been found to date. Therefore, prevention of dementia is a priority goal for the society. Since the groundbreaking description of a case report by Alois Alzheimer at the beginning of the twentieth century, dementia has been described in the early 1980s as "The Silent Epidemic," and, due to longer life and the huge number of baby boomers reaching old age, this epidemic will keep growing worldwide. The encouraging epidemiologic results of a decreasing dementia prevalence due to improved lifestyle and disease prevention will not be able to substantially counteract the increasing trend. Balanced nutrition is one critical aspect of health consciousness with beneficial effects against dementia-together with physical exercise, cognitive engagement and social activities (Scarmeas et al. 2009b; Polidori 2012b). Nutrition, like dementia, is a complex process extremely difficult to discompose, factorize and address in its multiple components. Therefore, it is highly time- and money-consuming to expect significant protective effects by single micronutrient supplementation in cognitively impaired subjects with a neurodegenerative process ongoing since years. Like the several ongoing RCTs in MCI and AD listed above, the debate on oxidative stress and antioxidants (http://www.nature.com/scientifica merican/journal/v308/n2/full/scientificamerican0213-62. html) will remain rather less substantial unless the methodological approach is changed (Fig. 2). The strongest evidence derived from well-controlled studies is in favor of healthy dietary patterns including balanced nutrition and adequate antioxidant status. There is an urgent need that physicians and health professional actively motivate subjects, long before they become AD patients, on living a healthy lifestyle. 
There is strong recent evidence for a link between $\mathrm{AD}$ and vascular disorders and risk factors as well as for the role of oxidative stress, reactive oxygen and nitrogen species and redox imbalance as the main pathophysiological factors mediating neurodegeneration with vascular pathology in $\mathrm{AD}$. In the context of $\mathrm{AD}$ complexity, optimal nutrition can negatively influence $\mathrm{AD}$ risk also through vascular protection and genetic/epigenetic control. Epigenetic modifications have been recently recognized as critical mediators of environmental effects on physiological genetic function. DNA methylation and histone modifications are two of the most common epigenetic modifications, with changes in gene promoters able to enhance susceptibility to neurodegeneration in the presence of other predisposing factors such as APO genotype of single nucleotide polymorphisms (SNPs). A third type of epigenetic modifications potentially contributing to gene dysregulation and neurodegenerative diseases including $\mathrm{AD}$ is single-stranded transcribed noncoding RNAs. These are 19-25 nucleotides long and are called micro-RNAs (miRNAs). They are able to modify chromatin and repress translational activity. Redox metabolism and nutritional factors have been shown to be able to influence in several directions these three types of epigenetic modifications both in late-onset $\mathrm{AD}$ and in the vascular pathology associated with $\mathrm{AD}$. In $\mathrm{AD}$, several miRNAs are abnormally expressed, although it is not clear yet if this altered expression occurs prior to altered $A \beta$ processing or as a consequence (Leszek et al. 2012).

A third and last point deserving attention together with nutrition-neurodegeneration interactions and vascularredox-epigenetic control is the likely though not yet clarified interrelationship between major hypotheses of lateonset AD pathogenesis. These hypotheses share in common both coexistence of multiple-usually two-pathophysiological factors and rationale and scientific success. At the end of the 1990s, de La Torre (2000) proposed that advanced aging in the presence of a vascular risk factor may accelerate the achievement of a critical threshold of cerebral hypoperfusion (critically attained threshold of cerebral hypoperfusion, $\mathrm{CATCH}$ ). The $\mathrm{CATCH}$ may trigger regional brain microcirculatory disturbance thereby impairing the adequate delivery energy substrates for normal cerebral function. Impaired energy metabolism and hypoperfusion of the brain are related to oxidative stress and aberrant mitogenic changes in $\mathrm{AD}$ pathogenesis. $\mathrm{Zhu}$ et al. (2004) addressed the possibility that although either latter mechanism can independently serve as AD initiator, both are necessary to propagate disease pathogenesis. This was called the "two-hit hypotheses." In 2006, Professor Bruce N. Ames published a key article in PNAS explaining why the deficiency in several micronutrients is associated with chromosome breaks and disease in humans (Ames 2006a, b). The so-called triage theory states that the scarce micronutrients present in a status of hidden malnutrition are used for short-term survival in spite of long-term survival, so that when an additional insult comes later during the course of susceptible life, pathology occurs (Ames $2006 a, b)$. The "latent early life-associated regulation" (LEARn) model suggests that environmental stress-likely and often oxidative stress and/or nutritional imbalancemarks a gene which later during life and in the presence of a secondary trigger-likely and often vascular and/or inflammatory factors-will be expressed aberrantly causing overt pathology (Lahiri et al. 2008; Lahiri and Maloney 2010). The epigenetic oxidative redox shift (EORS) theory of aging proposes that sedentary behavior associated with age triggers an oxidized redox shift and impaired mitochondrial function inducing an epigenetic vicious circle (Brewer 2010). These theories and hypotheses, observed from a network point of view, may trace a new path for a comprehensive approach to AD mechanisms and therapy.

\section{References}

Agüero-Torres HKM, von Strauss E (2006) Rethinking dementia diagnoses in a population-based study: what is Alzheimer's disease and what is vascular dementia? A study from the Kungsholmen Project. Dement Geriatr Cogn Disord 22:244-249

Ames BN (2006a) Low micronutrient intake may accelerate the degenerative diseases of aging through allocation of scarce micronutrients by triage. Proc Natl Acad Sci USA 103: 17589-17594

Ames BN (2006b) Low micronutrient intake may accelerate the degenerative diseases of aging through allocation of scarce micronutrients by triage. Proc Natl Acad Sci USA 103: 17589-17594

Anlasik T, Sies H, Griffiths HR, Stahl W, Polidori MC (2005) Dietary habits are major determinants of the plasma antioxidant status in healthy elderly subjects. Br J Nutr 94:639-642

Barberger-Gateau P, Raffaitin C, Letenneur L, Berr C, Tzourio C, Dartigues JF, Alperovitch A (2007) Dietary patterns and risk of dementia. Neurology 69:1921-1930

Bayer-Carter JL, Green PS, Montine TJ, VanFossen B, Baker LD, Watson GS, Bonner LM, Callaghan M, Leverenz JB, Walter BK, Tsai E, Plymate SR, Postupna N, Wilkinson CW, Zhang J, Lampe J, Kahn SE, Craft S (2011) Diet intervention and cerebrospinal fluid biomarkers in amnestic mild cognitive impairment. Arch Neurol 68(6):743-752

Boeing H, Bechthold A, Bub A, Ellinger S, Haller D, Kroke A, Leschik-Bonnet E, Müller MJ, Oberrittler H, Schulze M, Stehle P, Watzl B (2012) Critical review: vegetables and fruit in the prevention of chronic diseases. Eur J Nutr 51:637-663

Brewer GJ (2010) Epigenetic oxidative redox shift (EORS) theory of aging unifies the free radical and insulin signaling theories. Exp Gerontol 45:173-179

Burgener SC, Buettner L, Coen Buckwalter K, Beattie E, Bossen AL, Fick DM, Fitzsimmons S, Kolanowski A, Richeson NE, Rose K, Schreiner A, Pringle Specht JK, Testad I, Yu F, McKenzie S (2008) Evidence supporting nutritional interventions for persons in early stage Alzheimer's disease. J Nutr Health Aging 12:18-21 
Dai Q, Borenstein AR, Wu Y, Jackson JC, Larson EB (2006) Fruit and vegetable juices and Alzheimer's disease: the Kame Project. Am J Med 119(9):751-759

de la Torre JC (2000) Critically attained threshold of cerebral hypoperfusion: the CATCH hypothesis of Alzheimer's pathogenesis. Neurobiol Aging 21:331-342

de la Torre JC (2013) Vascular risk factors: a ticking time bomb to Alzheimer's disease. Am J Alzheimers Dis Other Dement 28:551-559

de Lorgeril M, Salen P, Martin JL, Monjaud I, Delaye J, Mamelle N (1999) Mediterranean diet, traditional risk factors, and the rate of cardiovascular complications after myocardial infarction: final report of the Lyon Diet Heart Study. Circulation 99(6):779-785

Dumont M, Lin MT, Beal MF (2010) Mitochondria and antioxidant targeted therapeutic strategies for Alzheimer's disease. J Alzheimers Dis 20:S633-S643

Elmadfa I (ed) (2009) European nutrition and health report 2009. Karger, Basel

Engelhart MJ, Geerlings MI, Ruitenberg A, van Swieten JC, Hoffman A, Witteman JC, Breteler MM (2002) Dietary intake of antioxidants and risk of Alzheimer's disease. JAMA 287:3223-3229

Feng-Jiao L, Shen L, Ji HF (2012) Dietary intakes of vitamin E, vitamin $\mathrm{C}$ and $\beta$-carotene and risk of Alzheimer's disease: a meta-analysis. J Alzheimer's Dis 31:253-258

Friedland RP (2003) Fish consumption and the risk of Alzheimer disease: is it time to make dietary recommendations? Arch Neurol 60(7):923-924

Grodstein F, Chen J, Willett WC (2003) High-dose antioxidant supplements and cognitive function in community-dwelling elderly women. Am J Clin Nutr 77:975-984

Hensley K, Benaksas EJ, Bolli R, Comp P, Grammas P, Hamdheydari L, Mou S, Pye QN, Stoddard MF, Wallis G, Williamson KS, West M, Wechter WJ, Floyd RA (2004) New perspectives on vitamin E: gamma-tocopherol and carboxyethylhydroxychroman metabolites in biology and medicine. Free Radic Biol Med 36:1-15

Hughes TF, Andel R, Small BJ et al (2010) Midlife fruit and vegetable consumption and risk of dementia in later life in Swedish twins. Am J Geriatr Psychiatry 18:413-420

Hughes TM, Rosano C, Evand RW, Kuller LH (2013) Brain cholesterol metabolism, oxysterols, and dementia. J Alzheimer's Dis 33:891-911

Joshi JB, Praticó D (2012) Vitamin E in aging, dementia, and Alzheimer's disease. BioFactors 38:90-97

Knoops KT, de Groot LC, Kromhout D, Perrin AE, Moreiras-Varela O, Menotti A, van Staveren WA (2004) Mediterranean diet, lifestyle factors, and 10-year mortality in elderly European men and women: the HALE project. JAMA 292(12):1433-1439

Kris-Etherton P, Eckel RH, Howard BV, Jeor S, Bazzarre TL (2001) Lyon Diet Heart Study: benefits of a Mediterranean-Style, National Cholesterol education program/American Heart Association Step I dietary pattern on cardiovascular disease. Circulation 103:1823-1825

Lahiri DK, Maloney B (2010) The "LEARn" (latent early-life associated regulation) model integrates environmental risk factors and the developmental basis of Alzheimer's disease, and proposes remedial steps. Exp Gerontol 45(4):291-296

Lahiri DK, Zawia NH, Greig NH, Sambamurti K, Maloney B (2008) Early-life events may trigger biochemical pathways for Alzheimer's disease: the "LEARn" model. Biogerontology 9(6):375-379

Lambrinoudaki I, Ceasu I, Depypere H, Erel T, Rees M, SchenckGustafsson K, Simoncini T, Tremollieres F, van der Schouw YT, Pérez-López FR (2013) EMAS position statement: diet and health in midlife and beyond. Maturitas 74:99-104
Larrieu S, Latenneur L, Helmer C, Dartigues JF, Barberger-Gateau P (2004) Nutritional factors and risk of incident dementia in the PAQUID longitudinal cohort. J Nutr Health Aging 8(3):150-154

Larson EB, Yaffe K, Langa KM (2013) New insights into the dementia epidemic. N Engl J Med 369:2275-2277

Leszek J, Sochocka M, Gasiorowski K (2012) Vascular factors and epigenetic modifications in the pathogenesis of Alzheimer's disease. J Neurol Sci 323:25-32

Leuner K, Müller WE, Reichert AS (2012) From mitochondrial dysfunction to amyloid beta formation: novel insights into the pathogenesis of Alzheimer's disease. Mol Neurobiol 46:186-193

Luchsinger JA, Wang MX, Shea S, Mayeux R (2003) Antioxidant vitamin intake and risk of Alzheimer disease. Arch Neurol 60:203-208

Mangialasche F, Solomon A, Winblad B, Mecocci P, Kivipelto M (2010) Alzheimer's disease: clinical trials and drug development. Lancet Neurol 9:702-716

Martínez-González MA, de la Fuente-Arrillaga C, Nunez-Cordoba JM, Basterra-Gortari FJ, Beunza JJ, Vazquez Z, Benito S, Tortosa A, Bes-Rastrollo M (2008) Adherence to Mediterranean diet and risk of developing diabetes: prospective cohort study. BMJ 336(7657):1348-1351

Masaki KH, Losonczy KG, Izmirlian G, Foley DJ, Ross GW, Petrovitch H, Havlik R, White LR (2000) Association of vitamin $\mathrm{E}$ and $\mathrm{C}$ supplement use with cognitive function and dementia in elderly men. Neurology 54:1265-1272

Mecocci P, Polidori MC (2012) Antioxidant clinical trials in mild cognitive impairment and Alzheimer's disease. Biochim Biophys Acta 1822:631-638

Morris MC, Evans DA, Bienias JL, Tangney CC, Bennett DA, Aggarwal N, Wilson RS, Scherr PA (2002) Dietary intake of antioxidant nutrients and the risk of incident Alzheimer disease in a biracial community study. JAMA 287:3230-3237

Morris MC, Evans DA, Tangney CC, Bienias JL, Wilson RS, Aggarwal NT, Scherr PA (2005a) Relation of the tocopherol forms to incident Alzheimer disease and to cognitive change. Am J Clin Nutr 81:508-514

Morris MC, Evans DA, Tangney CC, Bienias JL, Wilson RS, Aggarwal NT, Scherr PA (2005b) Relation of the tocopherol forms to incident Alzheimer disease and to cognitive change. Am J Clin Nutr 81:508-514

Morris MC, Evans DA, Tangney CC, Bienias JL, Wilson RS (2006) Associations of vegetable and fruit consumption with age-related cognitive change. Neurology 67:1370-1376

Petersen RC, Thomas RG, Grundman M, Bennett D, Doody R, Ferris S, Galasko D, Jin S, Kaye J, Levey A, Pfeiffer E, Sano M, van Dyck CH, Thal LJ (2005) Vitamin E and donepezil for the treatment of mild cognitive impairment. $\mathrm{N}$ Engl J Med 52:2379-2388

Polidori MC (2012a) Biofactors and cognitive function. BioFactors 38:77-171

Polidori MC (2012b) Demenz. Z Gerontol Geriatr Eur J Geriatr 45:4-54

Polidori MC, Pientka L (2012) Bridging the pathophysiology of Alzheimer's disease with vascular pathology: the feed-back, the feed-forward and oxidative stress. J Alzheimer's Dis 28:1-9

Polidori MC, Nelles G (2013) Antioxidant clinical trials in mild cognitive impairment and Alzheimer's disease-challenges and perspectives. Curr Pharmachol Des 20 (Epub ahead of print)

Polidori MC, Praticó D, Mangialasche F, Mariani E, Aust O, Anlasik T, Mang N, Pientka L, Stahl W, Sies H, Mecocci P, Nelles G (2009a) High fruit and vegetable intake is positively correlated with antioxidant status and cognitive performance in healthy subjects. J Alzheimers Dis 17:921-927

Polidori MC, Carrillo JC, Verde PE, Stahl W, Sies H, Siegrist J (2009b) Plasma micronutrient status is improved after a 3-month 
dietary intervention with 5 daily portions of fruits and vegetables: implications for optimal antioxidant levels. Nutr J 8:10-11

Polidori MC, Pientka L, Mecocci P (2012) A review of the major vascular risk factors related to Alzheimer's disease. J Alzheimer's Dis 32:521-530

Pomerleau J, McKee M, Lobstein T, Knai C (2003) The burden of disease attributable to nutrition in Europe. Public Health Nutr 6:453-461

Praticó D, Clark CM, Liun F, Rokach J, Lee VY, Trojanowski JQ (2002) Increase of brain oxidative stress in mild cognitive impairment: a possible predictor of Alzheimer's disease. Arch Neurol 59:972-976

Prince M, Bryce R, Albanese E, Wimo A, Ribeiro W, Ferri CP (2013) The global prevalence of dementia: a systematic review and metaanalysis. Alzheimers Dement 9:63-75

Rinaldi P, Polidori MC, Metastasio A, Mariani E, Mattioli P, Cherubini A, Catani M, Cecchetti R, Senin U, Mecocci P (2003) Plasma antioxidants are similarly depleted in mild cognitive impairment and in Alzheimer's disease. Neurobiol Aging 24:915-919

Ritchie K, Carriere I, Ritchie CW et al (2010) Designing prevention programmes to reduce incidence of dementia: prospective cohort study of modifiable risk factors. BMJ 341:c3885

Sano M, Ernesto C, Thomas RG, Klauber MR, Schafer K, Grundman M, Woodbury P, Growdon J, Cotman CW, Pfeiffer E, Schneider LS, Thal LJ (1997) A controlled trial of selegiline, alphatocopherol, or both as treatment for Alzheimer's disease. The Alzheimer's Disease Cooperative Study. N Engl J Med 336(17):1216-1222

Scarmeas N, Stern YY, Tang MX et al (2006) Mediterranean diet and risk for Alzheimer's disease. Ann Neurol 59:912-921

Scarmeas N, Stern Y, Meyeaux R et al (2009a) Mediterranean diet and mild cognitive impairment. Arch Neurol 66:216-225

Scarmeas N, Luchsinger JA, Schupf N, Brickman AM, Cosentino S, Tang MX, Stern Y (2009b) Physical activity, diet, and risk of Alzhemer's disease. JAMA 302:627-637
Schrijvers EMC, Verhaaren BFJ, Koudstaal PJ, Hofman A, Ikram MA, Breteler MMB (2012) Is dementia incidence declining? Trends in dementia incidence since 1990 in the Rotterdam Study. Neurology 78:1456-1463

Sies H (1985) Oxidative stress: introductory remarks. In: Sies H (ed) Oxidative stress. Academic Press, Orlando, pp 1-15

Sies H (1993) Strategies of antioxidant defense. Eur J Biochem 215:213-219

Smith MA, Nunomura A, Lee H, Zhu X, Moreira PI, Avila J, Perry G (2005) Chronological primacy of oxidative stress in Alzheimer's disease. Neurobiol Aging 26:579-580

Sofi F, Cesari F, Abbate R, Gensini GF, Casini A (2008) Adherence to Mediterranean diet and health status: meta-analysis. BMJ 337:A1344

Vagnucci AH, Li WW (2003) Alzheimer's disease and angiogenesis. Lancet 361:605-608

Whitehouse PJ, George DR (2011) Uncertain progress on the fuzzy boundaries of Alzheimer's disease: reading between the guidelines. J Alzheimers Dis 26:1-5

Willett WC, Sacks F, Thricopoulou A, Drescher G, Ferro-Luzzi A, Helsing E, Trichopoulos D (1995) Mediterranean diet pyramid: a cultural model for healthy eating. Am J Clin Nutr 61(6 Suppl):1402S-1406S

Yaffe K, Clemons TE, McBee WL, Lindblad AS (2004) Impact of antioxidants, zinc and copper on cognition in the elderly: a randomized, controlled trial. Neurology 63:1705-1707

Yan MH, Wang X, Zhu X (2013) Mitochondrial defects and oxidative stress in Alzheimer disease and Parkinson disease. Free Radic Biol Med 62:90-101

Zandi PP, Anthony JC, Khachaturian AS, Stone SV, Gustafson D, Tschanz JT, Norton MC, Welsh-Bohmer KA, Breitner JC (2004) Reduced risk of Alzheimer disease in users of antioxidant vitamin supplements: the Cache County Study. Arch Neurol 61:82-88

Zhu X, Raina AK, Perry G, Smith MA (2004) Alzheimer's disease: the two-hit hypothesis. Lancet Neurol 3:219-226 